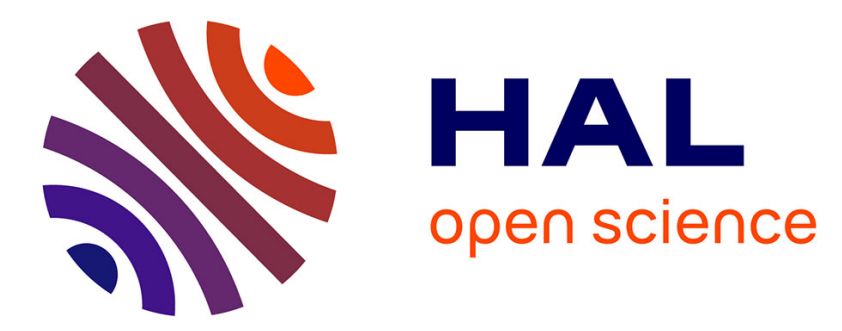

\title{
Spotting Handwritten Words and REGEX using a two stage BLSTM-HMM architecture
}

\author{
Gautier Bideault, Luc Mioulet, Clément Chatelain, Thierry Paquet
}

\section{To cite this version:}

Gautier Bideault, Luc Mioulet, Clément Chatelain, Thierry Paquet. Spotting Handwritten Words and REGEX using a two stage BLSTM-HMM architecture. Document Recognition and Retrieval, 2015, San Fransisco, United States. hal-01089168

\section{HAL Id: hal-01089168 https://hal.science/hal-01089168}

Submitted on 1 Dec 2014

HAL is a multi-disciplinary open access archive for the deposit and dissemination of scientific research documents, whether they are published or not. The documents may come from teaching and research institutions in France or abroad, or from public or private research centers.
L'archive ouverte pluridisciplinaire HAL, est destinée au dépôt et à la diffusion de documents scientifiques de niveau recherche, publiés ou non, émanant des établissements d'enseignement et de recherche français ou étrangers, des laboratoires publics ou privés. 


\title{
Spotting Handwritten Words and REGEX using a two stage BLSTM-HMM architecture
}

\author{
Gautier Bideault ${ }^{a}$, Luc Mioulet ${ }^{a}$, Clément Chatelain ${ }^{b}$ and Thierry Paquet $^{a}$ \\ ${ }^{a}$ Laboratoire LITIS - EA 4108, Universite de Rouen, FRANCE 76800; \\ ${ }^{b}$ Laboratoire LITIS - EA 4108, INSA Rouen, FRANCE 76800
}

\begin{abstract}
In this article, we propose a hybrid model for spotting words and regular expressions (REGEX) in handwritten documents. The model is made of the state-of-the-art BLSTM (Bidirectional Long Short Time Memory) neural network for recognizing and segmenting characters, coupled with a HMM to build line models able to spot the desired sequences. Experiments on the Rimes database show very promising results.
\end{abstract}

Keywords: Regular Expression Spotting, REGEX, Handwritten Document, Handwriting Recognition, BLSTM, Word Spotting

\section{INTRODUCTION}

Regular expression (REGEX) spotting consists in detecting patterns sequence of characters that obey certain

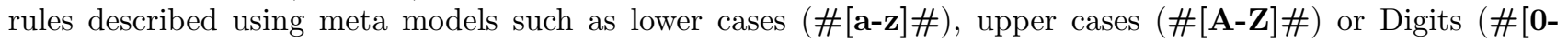
9]\#). Detecting such regular expression in handwritten documents can be useful for finding sub-string which are relevant for a further higher level information extraction task. For example, a system of that kind could spot entities. For example, spotting date $(\#[\mathbf{0 - 9}]\{\mathbf{2}\} /[\mathbf{0 - 9}]\{\mathbf{2}\} /[\mathbf{0 - 9}]\{\mathbf{4}\} \#)$, first name (\#[A-Z $\left.][\mathbf{a - z}]^{*} \#\right)$, ZIP code and city name of a french postal address $\left(\#[\mathbf{0 - 9}]\{\mathbf{5}\}[\mathbf{A}-\mathbf{Z}]^{*} \#\right)$. The extraction of these informations allow to consider high level processing stages such as document categorisation, customer identification, Named Entity detection, etc.

Some REGEX spotting systems have been designed for electronical documents, using Natural Language Processing methods. ${ }^{1,2}$ In this case, the REGEX spotting is rather straightforward as it consists in applying exact string matching methods on the ASCII text. When dealing with document images, a recognition step is needed in order to produce the ASCII transcription before processing the input data. The trouble is that this recognition step is subject to errors and uncertainty, making the string matching problematic. Some attempts have been made on printed documents. ${ }^{3,4}$ In these works, an OCR is applied on the whole document before applying the regular expression spotting step based on a set of rules that performs an exact matching. In spite of OCR errors, the system provides acceptable performance*.

To the best of our knowledge, there are only a few works concerning REGEX spotting in handwritten documents. The reason is that exact matching methods can not overcome the frequent recognition errors due to the intrinsic difficulties of recognizing handwriting. Therefore, in order to cope with these errors, inexact matching method should be carried out. This can be performed using statistical sequence models such as HMM. Some works have been published within this framework, proposing pattern spotting such as dates ${ }^{5}$ and numerical fields ${ }^{6,7}$ that involve meta models of characters, namely digits. However, these HMM based approach are limited to very specific fields. A more generic approach for REGEX spotting in handwritten documents has been addressed using pure HMM approach ${ }^{8}$, but led to moderate results (see section 5 for detailed results and comparison).

In this paper, a REGEX spotting system for handwritten documents is proposed based on a combination of HMM statistical sequence model with the state-of-the-art BLSTM neural network. Our alternative hybrid BLSTM/HMM model enables us to benefit from both strong local discrimination, and the generative sequence ability of the HMM.

\footnotetext{
${ }^{*}$ Average Precision of $82 \%$ and $72 \%$ of Recall
} 
This paper is organized as follows: first a review of word and REGEX spotting is given in section 2, then we present our REGEX spotting system based on a hybrid BLSTM/HMM in section 3. Section 4 is devoted to the experimental setup and results on both word spotting and regular expression spotting tasks carried out on the RIMES database. ${ }^{9}$

\section{RELATED WORK}

As a REGEX can match sequences with variable length and characters, a REGEX spotting task can be assimilated to a word spotting task where the word belongs to a lexicon which contains all the character string variations admissible by the REGEX. The less constrained the REGEX, the larger the size of the lexicon. Relaxing those constraints makes the REGEX spotting task more complex especially when considering handwritten document images. As regular expression spotting shares many aspects in common with word spotting we now briefly introduce the related works concerning word spotting approaches.

Word spotting in document images has received a lot of attention these last years. Systems proposed in the literature are divided into two main categories : Image based and recognition based systems. The first one, also known as query-by-example, operates through the image representation of the keywords. ${ }^{10-14}$ Such systems are therefore limited to deal with omni-writer handwriting and require to get an image of the query. The second kind of approaches, also known as query-by-string methods, deals with the ASCII representation of the keywords. ${ }^{15-19}$ Moving from the image representation to the ascii representation of the query is performed through a recognition stage. These systems are suitable for omni-writer handwriting and can be used with any string query of any size. In this context, many works have focused on several variants of Hidden Markov Models (HMMs) to process this intrinsically sequential problem. ${ }^{19}$

State-of-the-art recognition-based approaches are based on a line of text models. ${ }^{17-19}$ The line model generally contains a model of the target word, combined with filler models that describe the out-of-vocabulary words. For example in Thomas et al., ${ }^{17}$ the authors present an alpha-numerical information extraction system on handwritten unconstrained documents. It relies on a global line modeling allowing a dual representation of the relevant and the irrelevant information. The acceptation or rejection of the matched keyword is controlled by the variation of a hyper-parameter in the HMM line model. A similar approach is presented in Fisher et al. ${ }^{18}$ The line model is made of a left and right filler models surrounding the word model. The acceptation or rejection of the matched keyword is controlled by a text line score based on the likelihood ratio of the word text line and the filler text line model. However we know that HMM rely on strong observation independence assumptions and they perform poorly on high dimensional observations. Moreover, they have low discrimination capabilities between character classes due to their inherently generative modelization framework.

Recently a new approach based on recurrent neural networks has overcome these shortcomings. Bilateral Long Short Term Memory (BLSTM) architecture has demonstrated impressive capabilities for omni-writer handwriting recognition. ${ }^{20}$ Some primary applications of BLSTM to word spotting have also demonstrated promising results. ${ }^{21,22}$ In this system, the BLSTM is combined with the CTC layer which provides character class posterior probabilities. Then a token passing algorithm allows efficient decoding of the spotting line model. Very interesting results have been reported on the IAM Database ${ }^{21 \dagger}$.

In this paper, we combine the BLSTM-CTC architecture with a HMM based spotting line model. This two stage architecture is first evaluated for hadnwritten word spotting on the RIMES database. Then we explore some extensions of the system to Regular Expression spotting (REGEX Spotting). This model is described in the following section.

\section{A TWO STAGE BLSTM-CTC HMM FOR INFORMATION SPOTTING}

In this section, we describe our hybrid model for word and REGEX spotting. We first describe the BLSTM-HMM architecture that has been retained, then we present our word spotting model, based on standard state-of-the-art word spotting framework. And finally, we propose the adaptation of this model for REGEX spotting.

\footnotetext{
${ }^{\dagger}$ Average Precision of $88.15 \%$ and R-precision of $84.34 \%$
} 


\subsection{BLSTM-CTC for character recognition and segmentation}

The BLSTM-CTC is a complex Recurrent Neural Network able to manage long term dependencies thanks to its internal buffer structure. Each neuron is specialized to stop a specific character in the input signal. The recurrent architecture allows each neuron to take account of the previous activated neurons (character), possibly at multiple time step earlier in the input signal (thus modeling long term dependencies). This typical architecture allows to take account of character bigrams in addition to the input signal to compute the activation of each neuron. The BLSTM is composed of two recurrent neural networks with Long Short Term Memory neural units. The first one processes the data from left to right whereas the second one proceeds in the reverse order. For each time step, decision is taken combining the two networks output, taking advantage of both left and right context. Such context is essential to have a certain knowledge of the surounding characters, because in most cases sequences of letters are constrained by the properties of the lexicon. The outputs of these two networks are then combined through a Softmax decision layer that provides character posterior probabilities in addition to a non decision class. This decision stage is called the Connectionist Temporal Classification (CTC $)^{23}$ that enables the labelling of unsegmented data.

These networks integrate special neural network units : Long Short Term Memory ${ }^{23}$ (LSTM). LSTM neurons is composed of a memory cel, an input and three control gates. Each gates control the memory of the cell, i.e how a given input will affect the memory (input gates), if a new input should reset the memory cell (forget gate) and if the memory of the network should be presented to the following neuron (output gate). This system of control gates allows a very accurate control of the memory cell during the training step. A LSTM layer is fully recurrent, that is to say, the input and the three gates receive at each instant $t$ the input signal at time $t$ and the previous outputs (at time $(t-1)$ ).

This architecture has shown very impressive results on challenging data-sets dedicated to word recognition ${ }^{9,24}$ due to its efficient classification and segmentation ability. For these reasons, its efficiency to cope with the low level character identification appears also very promising for handwritten words and REGEX spotting, since such scenario is less contrained by lexicon properties.

The proposed BLSTM/HMM architecture has been chosen in order to take advantage of both generative and discriminative frameworks. As shown on Figure 3.1, the input sequence is processed by a BLSTM-CTC network in order to compte character posterior probabilities at every step. Then the probabilities of each labels are fed to the HMM stage (using class posteriors in place of the character likelihood computed by Gaussian Mixtures Models in the traditionnal HMM framework) to perform the alignment of the spotting model. We now describe the HMM line spotting models which enable us to spot either words (cf section 3.2) or REGEX (cf section 3.3).

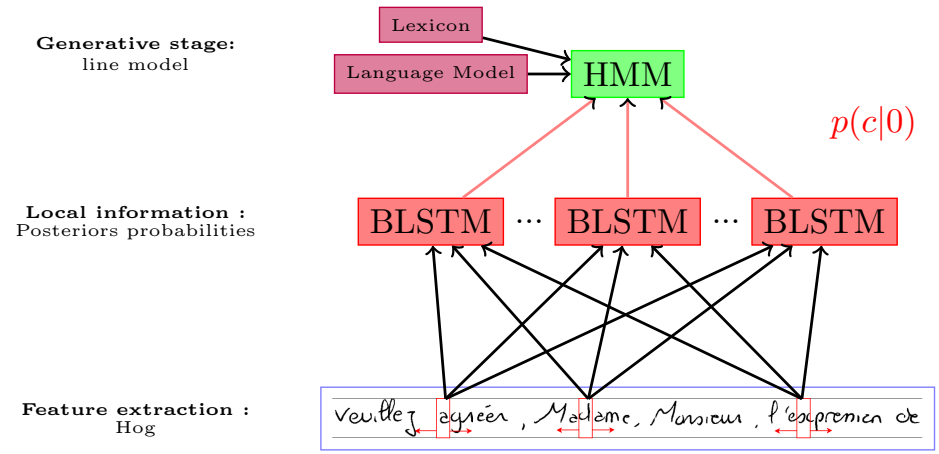

Figure 1. Hybrid structure BLSTM/HMM : Detail of the communication between both stages. The BLSTM/CTC outputs a posteriori probabilities for HMM decoding. 


\subsection{Handwritten word spotting model}

Our word spotting model describes a line of text that may contain the word to spot. As it is classically proposed in the literature, it is made of the HMM word model surrounded by filler models that represent any other sequence of characters. Figure 2 shows an example of a word spotting model for the word "Madame". The space model is directly integrated into the filler. By constraining the whole model, we can locate the word at the beginning, in the middle or at the end of the line. The filler model is basically an ergodic model made of every character model. In our problem, we use 99 models corresponding to lower and upper cases, digits, punctuations and space.

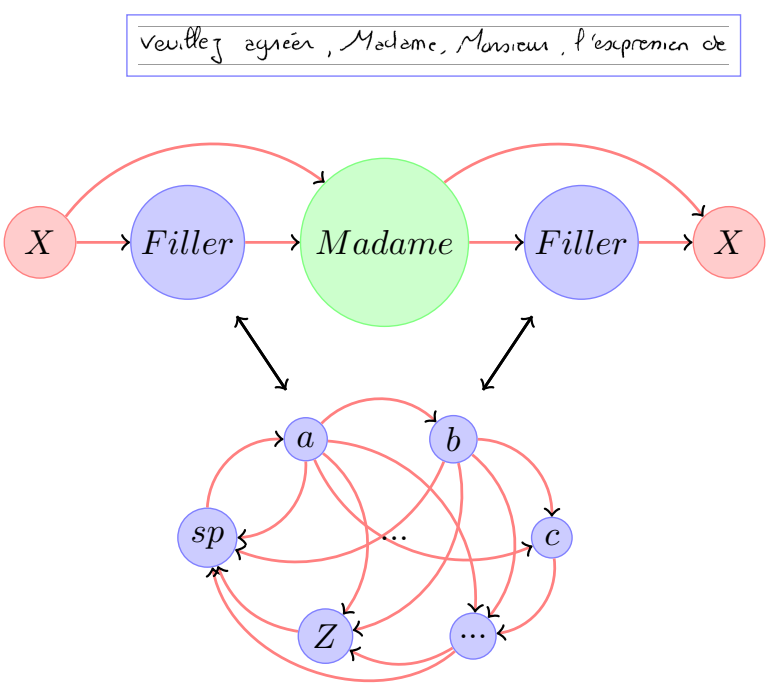

Figure 2. HMM line model : Detail of every component of the line model

Decoding a text line is classically achieved using the Viterbi algorithm, the system will outputs the character sequence with the maximum likelihood $P(X \mid \lambda)$. In order to accept the spotted word or reject it, decoding is generally performed twice: a first pass using the spotting model, and a second pass using a filler model. The likelihood ratio of the two models serves generally as a score for accepting or rejecting the spotted hypothesis. Using the BLSTM-CTC architecture, posterior probabilities are computed that can directly serve as a score for accepting/rejecting the hypothesis, without the need for a filler model. The score of each spotted hypothesis is computed by the average character posteriors over the number of frames spanning the hypothesis. This score is then normalised by the number of characters of the spotted word. Doing this, we choose to rely on the strong discriminative decisions of the BLSTM-CTC and use the HMM only as a sequence model constrained by high level information such as lexicons and/or language models. The graphical representation of the whole word spotting system is shown on Figure 3

We now show how this model can be adapted to REGEX spotting.

\subsection{Regular expression spotting model}

As previously mentionned, REGEX spotting is a generalisation of the word spotting task, the difference is that the sequences to spot are less constrained and more variable, thus leading to a larger lexicon of admissible expressions.

In order to cope with REGEX queries, we use the HMM stage to model a regular expression with a stochastic model of character sequences. Each meta model is an ergodic model of characters implied in the query, e.g, 


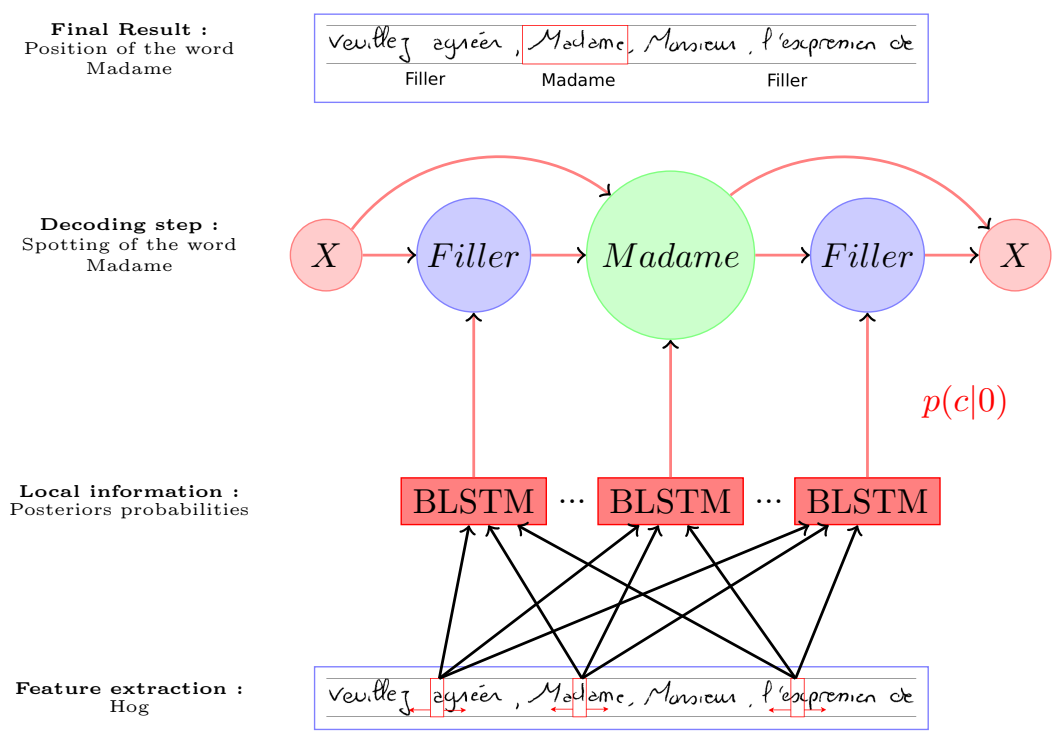

Figure 3. Hybrid structure BLSTM/HMM : Details of every step of the word spotting task from feature extraction to position of the word Madame in the sentence.

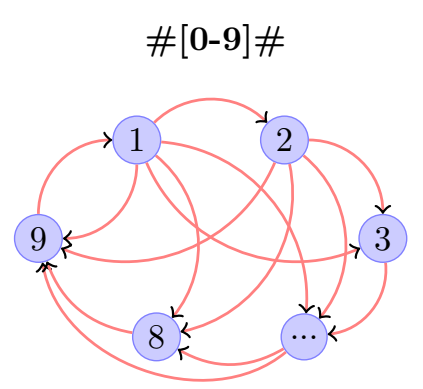

Digits MetaModel (DG)

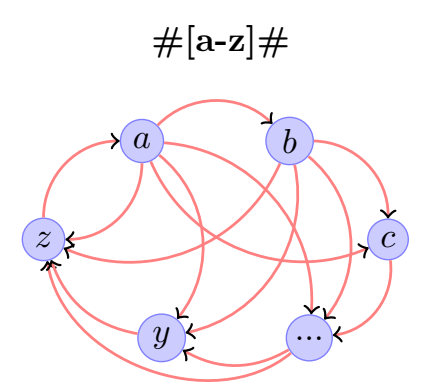

Lower Cases MetaModel (LC) Upper Cases MetaModel (UC)

Figure 4. HMM MetaModels 


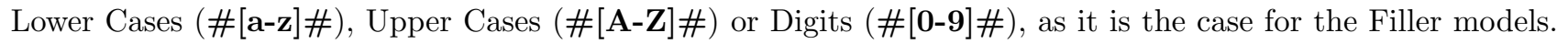
Figure 4 shows examples of meta models for these three examples.

We also need to model the variable length of the queries, which may occurs when using $*$ or + operators (spotting between 0 and $\infty$ times a character, or spotting between 1 and $\infty$ times a character) such as in \#[09]+\# which stands for any sequence of at least 1 digit. This is simply modeled by allowing auto transitions over the desired character meta model. Figure 5 shows an example of a model for spotting variable length sequences. The query taken is the sub-string agr following by an unconstrained sequence of lower cases $\left(\#[\mathbf{a}-\mathbf{z}]^{*} \#\right)$, in this example we hope that the system will spot the word agréer correctly.

The following models allow searching for a REGEX at the beginning of a line (\#[a-z] ${ }^{*}$ ion\#), at the end of a line $\left(\left(\# \mathbf{l e}[\mathbf{a}-\mathbf{z}]^{*} \#\right)\right)$, or both $(\#[\mathbf{A}-\mathbf{Z}] \mathbf{o}[\mathbf{a}-\mathbf{z}] \#)$. The line model can also only contain meta models dedicated to spotting sequences of digits of any length, for example $\left(\#[\mathbf{0 - 9}]^{*} \#\right)$ or word beginning by one upper case character and ending with a sequence of lower cases characters of arbitrary length $\left(\#[\mathbf{A}-\mathbf{Z}][\mathbf{a}-\mathbf{z}]^{*} \#\right)$. Here, the arbitrary length of the sequence unconstrained $\left(^{*}\right)$ is controled by the auto-transition probabilities of the meta model of the HMM.

Vevillez agreér, Madame, Monsieur, l'exprenien de

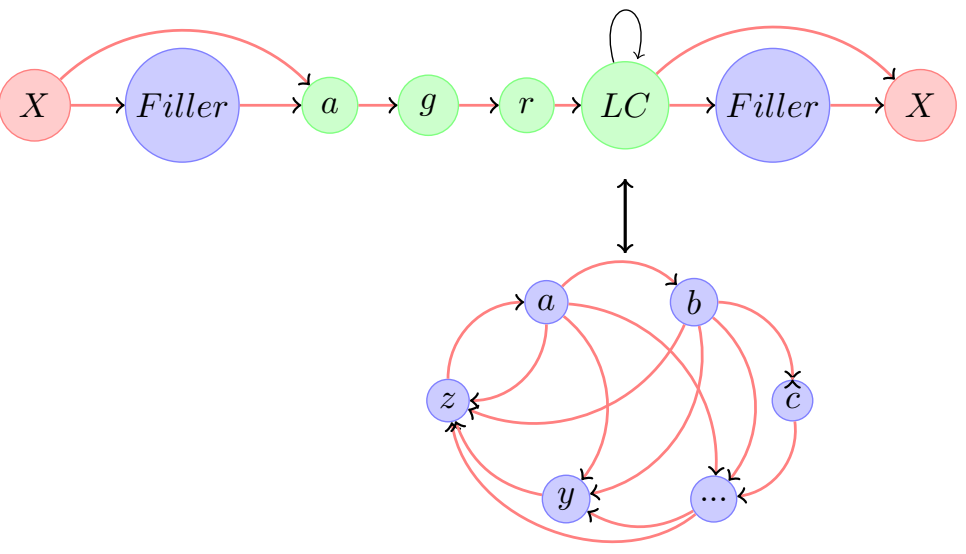

Figure 5. HMM stage : Spotting of regular expressions \#agr[a-z] \# (i.e every word beginning by the sub-string agr followed by any number of lower case characters)

As the transitions in the HMM meta models are ergodic, the Viterbi alignment will only be driven by the local classification of BLSTM-CTC. The spotting model depends on its discriminant capacity to feed the higher HMM stage with accurate information from the local character recognition stage.

The graphical representation of the whole REGEX spotting system is shown on Figure 6.

Finally, the integration of meta models and auto transitions into the line model allows spotting of handwritten REGEX. Practically, the line model is build on the fly at the time of querying the data-set, by rewriting the REGEX into a HMM line spotting model. At this time, the "translation" is manually done, but an automatization of this task can be performed for industrial purpose.

\section{EXPERIMENTS}

In this section, we give some details about the implementation of the system, starting with a description of the preprocessing stage in section 4.1 and the features extraction in section 4.2. The performance of the system are 


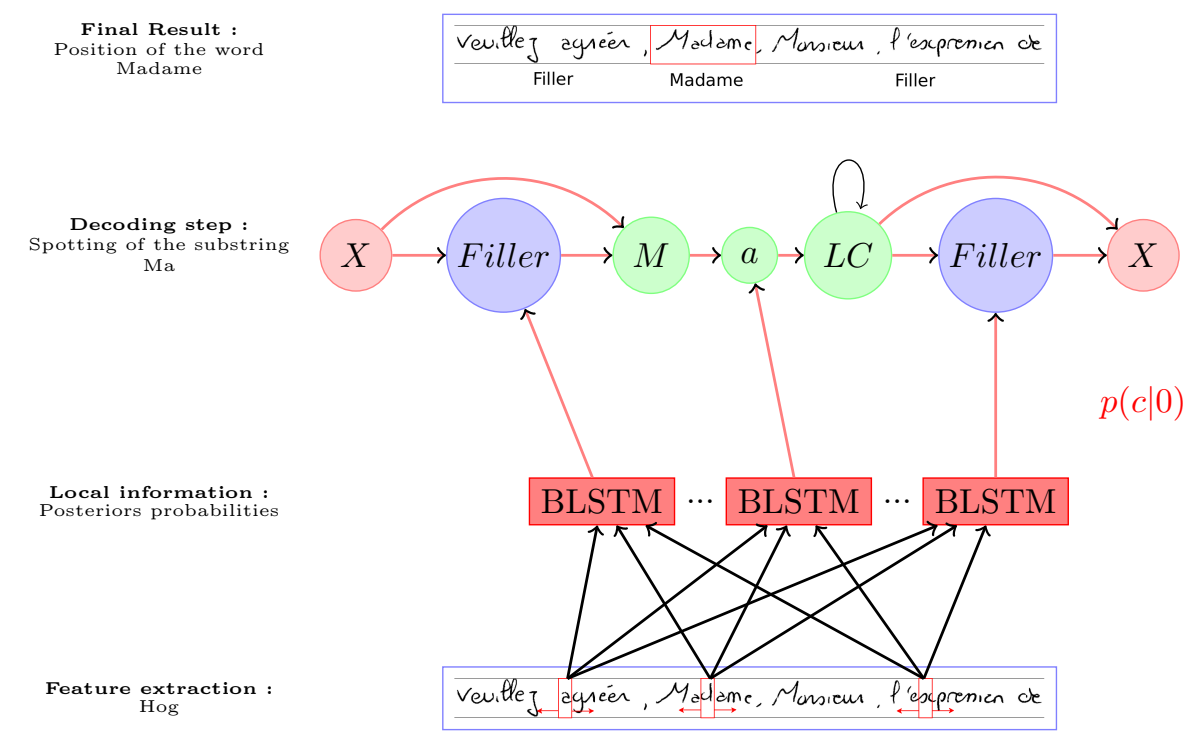

Figure 6. Hybrid structure BLSTM/HMM : Details of every step of the REGEX spotting task from feature extraction to position of the REGEX \# Ma[a-z]* \# in the sentence.

evaluated using the 2011 RIMES database ${ }^{25}$ they are summarized in section 4.3 .

\subsection{Details of Preprocessing}

The preprocessing of images consist in a simple three step image correction over the whole line. First a Sauvola binarisation by thresholding is applied. Then a deslanting process is carried out according to the following method: the image is rotated between $[-\Theta ; \Theta]$ and for each angle the histogram of vertical continuous traits $H_{\Theta}$ is measured. Finally, a height normalisation is applied to center the baseline and normalize the heights of the ascenders and descenders.

\subsection{Features Set}

Our feature vector is based on Histograms of Oriented Gradient (HOG) ${ }^{26}$ extracted from windows of $8 \times 64$ pixels. During the extraction, the window is dividing into sub-windows of $n \times m$ pixels. For each sub-window a histogram is computed, representing the distribution of the local intensity gradients (edge direction). The histograms of every windows are then merged to obtain the final representation of our feature vector representation. We used $8 \times 8$ non-overlapping sub-windows using 8 directions, this produces a 64 dimensional feature vector.

\subsection{Results and discussion}

To evaluate the performance of our system, all the experiments have been performed on the RIMES database used for the 2011 ICDAR handwriting recognition competitions. ${ }^{25}$ The training database is composed of 1.500 documents, the validation and test sets are composed respectively of 100 documents. In order to evaluate the spotting system, we compute recall $(\mathrm{R})$ and precision measures $(\mathrm{P})$. To do this, the number of true positives (TP), false positives (FP), and false negatives (FN) are evaluated for all possible threshold values. From these values, a recall-precision curve is computed by accumulating these values over all word queries.

$$
R=\frac{T P}{T P+F N} P=\frac{T P}{T P+F P}
$$




\subsubsection{Word spotting results}

Word spotting systems are generally evaluated by spotting one word in a collection of documents. We have decided to evaluate our framework by searching a set of words (a lexicon) at the same time, allowing to deal with confusion between words. ${ }^{8}$ Spotting a lexicon of keyword instead of a single word is well-suited for document categorization. ${ }^{27}$ We evaluate the performance of our system on lexicon of size 25,50 and 100 words respectively, following the same data-set and protocol as in Kessentini et al. ${ }^{8}$ The recall-precision curves are obtained by varying the threshold applied on line scores (see section 3.2).

Figure 7 presents the recall-precision curves obtained by our BLSTM-HMM hybrid model for each lexicon (25, 50 and 100). It is compared with the results obtained by the standard HMM method developed in Kessentini et $a l .{ }^{8}$ As expected, the performance drop when the size of the lexicon increase. The BLSTM/HMM provides very interesting results, since the mean average precision ${ }^{\ddagger}$ is greater than $80 \%$ for 25 -lexicon and 50 -lexicon queries. When compared with the pure HMM approach, results show that adding the discriminative stage has drastically improve the performance of the whole system. Indeed, we can observe a significant gap of at least $20 \%$ between the mean average precision of the two systems, for each lexicon size. This is certainly due to the fact, that the local decision of the BLSTM are far more discriminative than Gaussian Mixtures.

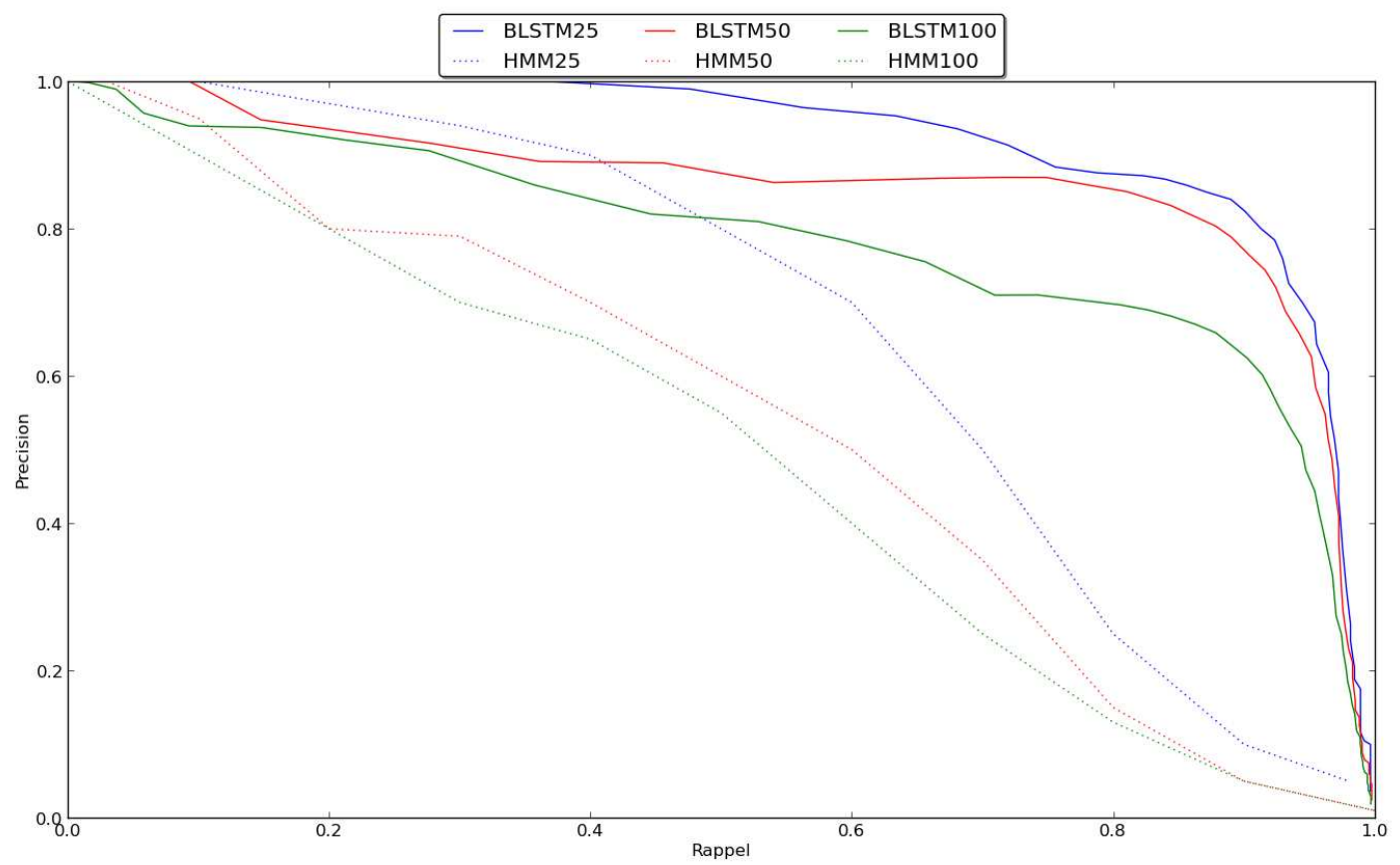

Figure 7 . Word spotting performance with different lexicons of words

\subsubsection{Regular expression results}

To evaluate the performance of our system on a regular expression spotting task we performed exactly the same experiments as in. ${ }^{8}$ In this study the authors were interested in spotting 4 different REGEX queries corresponding to the the search for the sub-strings "effe", "pa", "com" and "cha" at the beginning of a word (\#effe[a-z]*\#, $\left.\# \mathbf{p a}[\mathbf{a}-\mathbf{z}]^{*} \#, \# \operatorname{com}[\mathbf{a}-\mathbf{z}]^{*} \#, \# \mathbf{c h a}[\mathbf{a}-\mathbf{z}]^{*} \#\right)$. As for word spotting experiments, results of the HMM system have been added too in order to provide a precise comparison between those systems (cf Figure 8).

A first observation is that the system achieves good performance, since most of the REGEX queries lead to a mean-average precision of nearly $75 \%$, whereas the queries involve many fewer constraints than for word spotting. Moreover, our results are far beyond the standard HMM approach. We can observe a gap of more

\footnotetext{
${ }^{\ddagger}$ or break-even point: the point where Recall=Precision
} 
than $40 \%$ in the difficult cases $\left(\# \operatorname{com}[\mathbf{a}-\mathbf{z}]^{*} \#\right)$ and $\left(\# \mathbf{c h a}[\mathbf{a}-\mathbf{z}]^{*} \#\right)$ and $20 \%$ in easier ones $\left(\#\right.$ effe $[\mathbf{a}-\mathbf{z}]^{*} \#$ and $\left.\# \mathbf{p a}[\mathbf{a}-\mathbf{z}]^{*} \#\right)$.

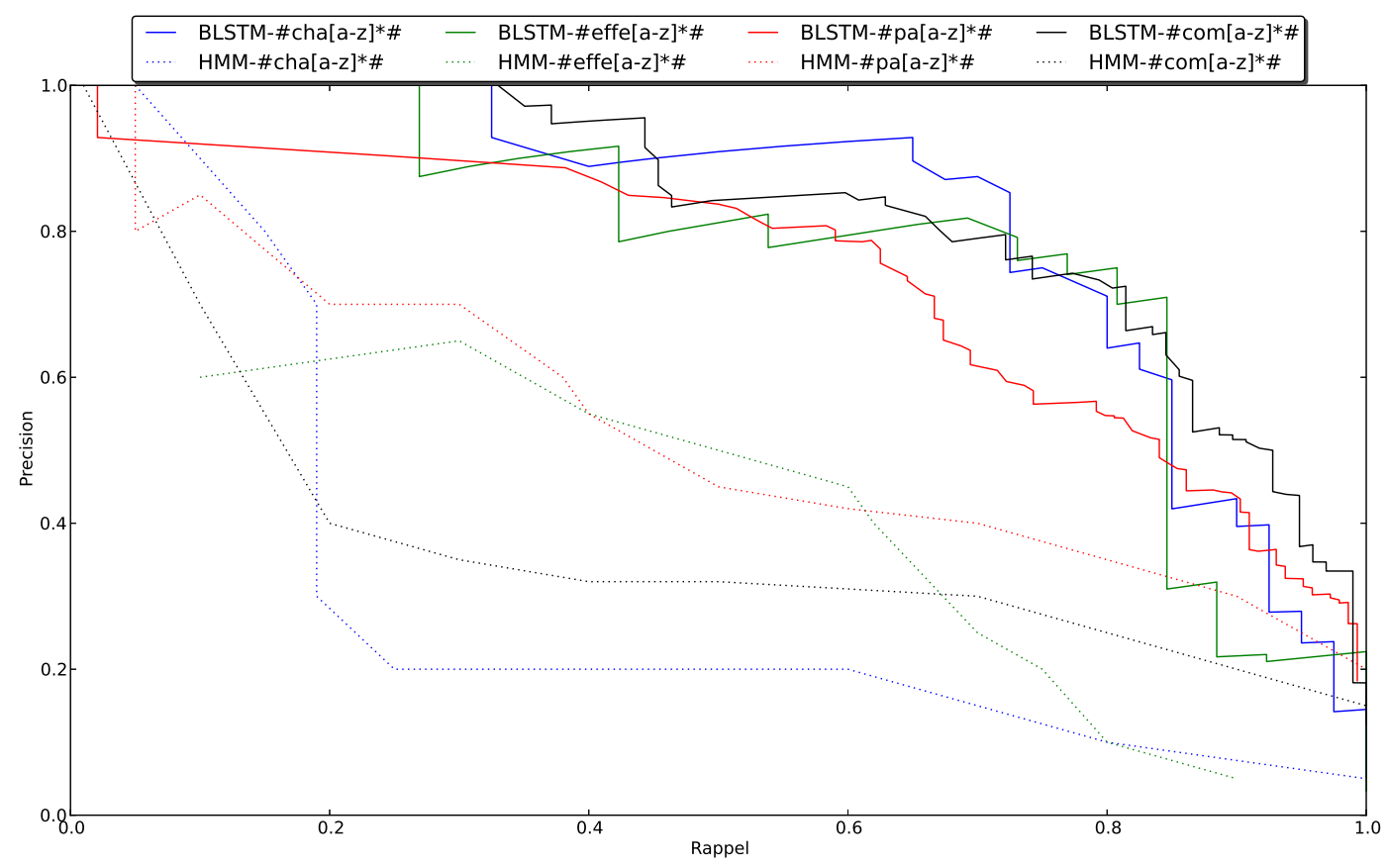

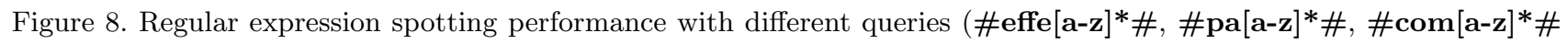
and \# cha $\left.[\mathbf{a - z}]^{*} \#\right)$

We have also tested less constrained queries, with the search for REGEX containing any sequence of upper cases characters $\left(\#[\mathbf{A}-\mathbf{Z}]^{*} \#\right)$, and any sequence of digits $(\#[\mathbf{0 - 9}] \#)$. This problem is by far more difficult than the previous queries since the corresponding sequences may have variable contents and lengths. For example the digit query should detect the sequence "1" as well as sequence "0123456789". Results are presented in Figure 9.

Knowing the difficulty of the problem, the performance are still interesting. Note that digit characters are not very frequent in the database. An interesting fact is that the Uppercase query can reach interesting precision scores, whereas the digit query can reach very high recall scores.

\section{CONCLUSION}

In this paper, we have proposed a hybrid system BLSTM-CTC/HMM able to spot any word of REGEX. We have shown that the hybrid system exhibits interesting results, even on weakly constrained queries such as the search for sequences of digits of arbitrary length. We have compared our system for REGEX spotting with some recent work carried out on the same data-set and using the standard HMM framework. Our approach outperforms this system by more than $30 \%$ on the standard word spotting task and by more than $40 \%$ on REGEX spotting. These very promising results allow to envisage the application of higher level spotting systems such as addresses, named entities for which a combination of specific markers (keywords and alpha numerical expressions) is generally used to detect the relevant information.

\section{REFERENCES}

[1] Dengel, A. R. and Klein, B., "smartfix: A requirements-driven system for document analysis and understanding," in [Document Analysis Systems V], 433-444, Springer (2002).

[2] Holzinger, W., Krüpl, B., and Herzog, M., [Using ontologies for extracting product features from web pages], Springer (2006). 


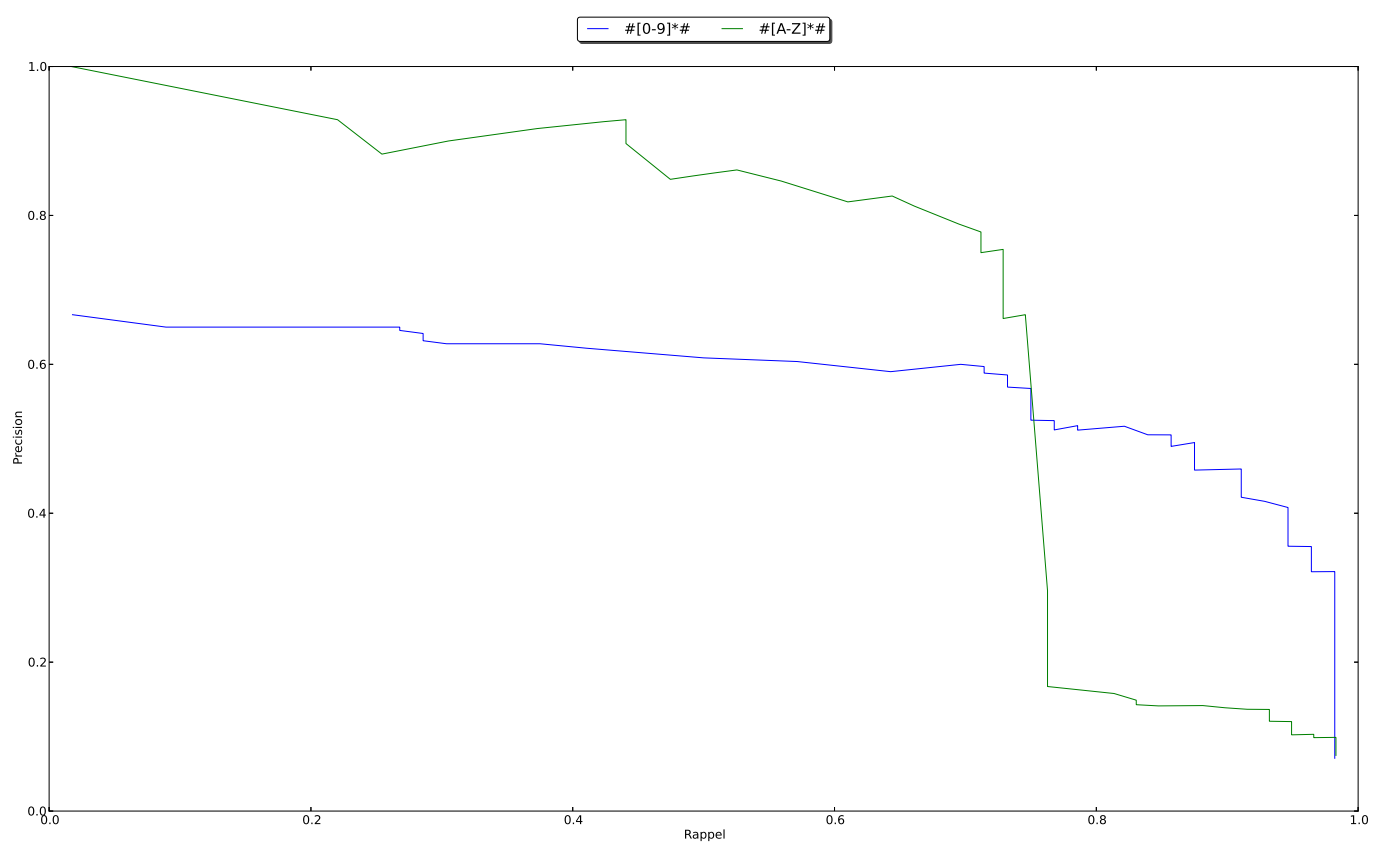

Figure 9. Regular expression spotting performance with upper case sequence $\left(\#[\mathbf{A}-\mathbf{Z}]^{*} \#\right)$ and number sequence $(\#[\mathbf{0}-$ 9]\#)

[3] Spitz, A., "Using character shape codes for word spotting in document images," in [In: Proceedings of the symposium on document analysis and information retrieval], 382-389 (1995).

[4] .Spitz, A., "Determination of script, language content of document images," in [IEEE Transactions on Pattern Analysis and Machine Intelligence], 19, 235-245 (1997).

[5] Morita, M. E., Sabourin, R., Bortolozzi, F., and Suen, C. Y., "Segmentation and recognition of handwritten dates: an hmm-mlp hybrid approach.," IJDAR , 248-262 (2003).

[6] Chatelain, C., Heutte, L., and Paquet, T., "A two-stage outlier rejection strategy for numerical field extraction in handwritten documents," in [ICPR, Hong Kong, China], 3, 224-227 (2006).

[7] Chatelain, C., Heutte, L., and Paquet, T., "Recognition-based vs syntax-directed models for numerical field extraction in handwritten documents," in [ICFHR, Montreal, Canada], 6p (2008).

[8] Kessentini, Y., Chatelain, C., and Paquet, T., "Word spotting and regular expression detection in handwritten documents," in $[I C D A R],(2013)$.

[9] Grosicki, E. and El Abed, H., "Icdar 2009 handwriting recognition competition," in [Document Analysis and Recognition, 2009. ICDAR'09. 10th International Conference on], 1398-1402, IEEE (2009).

[10] Rath, T. M. and Manmatha, R., "Features for word spotting in historical manuscripts," in [Document Analysis and Recognition, 2003. Proceedings. Seventh International Conference on], 218-222, IEEE (2003).

[11] Cao, H. and Govindaraju, V., "Template-free word spotting in low-quality manuscripts," in [Proceedings of the 6th International Conference on Advances in Pattern Recognition], 135-139 (2007).

[12] Adamek, T., OConnor, N. E., and Smeaton, A. F., "Word matching using single closed contours for indexing handwritten historical documents," International Journal of Document Analysis and Recognition (IJDAR) 9(2-4), 153-165 (2007).

[13] Rusinol, M., Aldavert, D., Toledo, R., and Lladós, J., "Browsing heterogeneous document collections by a segmentation-free word spotting method," in [Document Analysis and Recognition (ICDAR), 2011 International Conference on], 63-67, IEEE (2011). 
[14] Rodríguez-Serrano, J. A., Perronnin, F., Lladós, J., and Sánchez, G., "A similarity measure between vector sequences with application to handwritten word image retrieval," in [Computer Vision and Pattern Recognition, 2009. CVPR 2009. IEEE Conference on], 1722-1729, IEEE (2009).

[15] Rodríguez-Serrano, J. A. and Perronnin, F., "Handwritten word-spotting using hidden markov models and universal vocabularies," Pattern Recognition 42(9), 2106-2116 (2009).

[16] Frinken, V., Fischer, A., Manmatha, R., and Bunke, H., "A novel word spotting method based on recurrent neural networks," Pattern Analysis and Machine Intelligence, IEEE Transactions on 34(2), 211-224 (2012).

[17] Thomas, S., Chatelain, C., Heutte, L., and Paquet, T., "An information extraction model for unconstrained handwritten documents," in [Pattern Recognition (ICPR), 2010 20th International Conference on], 34123415, IEEE (2010).

[18] Fischer, A., Keller, A., Frinken, V., and Bunke, H., "Lexicon-free handwritten word spotting using character hmms," Pattern Recognition Letters 33(7), 934-942 (2012).

[19] Wshah, S., Kumar, G., and Govindaraju, V., "Script independent word spotting in offline handwritten documents based on hidden markov models," in [Frontiers in Handwriting Recognition (ICFHR), 2012 International Conference on], 14-19, IEEE (2012).

[20] Graves, A., Liwicki, M., Bunke, H., Schmidhuber, J., and Fernández, S., "Unconstrained on-line handwriting recognition with recurrent neural networks," in [Advances in Neural Information Processing Systems], 577584 (2008).

[21] Frinken, V., Fischer, A., and Bunke, H., "A novel word spotting algorithm using bidirectional long shortterm memory neural networks," in [Artificial Neural Networks in Pattern Recognition], Schwenker, F. and El Gayar, N., eds., Lecture Notes in Computer Science 5998, 185-196, Springer Berlin Heidelberg (2010).

[22] Wöllmer, M., Eyben, F., Graves, A., Schuller, B., and Rigoll, G., "A tandem blstm-dbn architecture for keyword spotting with enhanced context modeling," in [Proc. of NOLISP], (2009).

[23] Graves, A., Fernández, S., Gomez, F., and Schmidhuber, J., "Connectionist temporal classification: labelling unsegmented sequence data with recurrent neural networks," in [Proceedings of the 23rd international conference on Machine learning], 369-376, ACM (2006).

[24] Graves, A., Liwicki, M., Fernández, S., Bertolami, R., Bunke, H., and Schmidhuber, J., "A novel connectionist system for unconstrained handwriting recognition," Pattern Analysis and Machine Intelligence, IEEE Transactions on 31(5), 855-868 (2009).

[25] Grosicki, E. and El-Abed, H., "Icdar 2011-french handwriting recognition competition," in [Document Analysis and Recognition (ICDAR), 2011 International Conference on], 1459-1463, IEEE (2011).

[26] Rodriguez, J. A. and Perronnin, F., "Local gradient histogram features for word spotting in unconstrained handwritten documents," in [Int. Conf. on Frontiers in Handwriting Recognition], (2008).

[27] Paquet, T., Heutte, L., Koch, G., and Chatelain, C., "A categorization system for handwritten documents," International Journal on Document Analysis and Recognition 15(4), 315-330 (2012). 